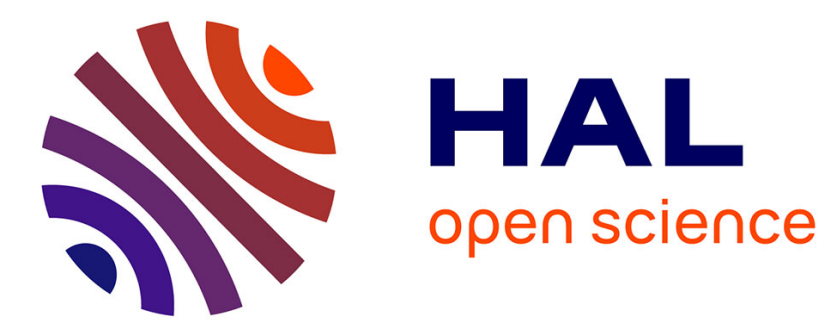

\title{
Robust damage assessment of multiple cracks based on the frequency response function and the Constitutive Relation Error updating method
}

Béatrice Faverjon, Jean-Jacques Sinou

\section{- To cite this version:}

Béatrice Faverjon, Jean-Jacques Sinou. Robust damage assessment of multiple cracks based on the frequency response function and the Constitutive Relation Error updating method. Journal of Sound and Vibration, 2008, 312, pp.821-837. 10.1016/j.jsv.2007.11.024 . hal-00322909

\section{HAL Id: hal-00322909 \\ https://hal.science/hal-00322909}

Submitted on 26 Sep 2012

HAL is a multi-disciplinary open access archive for the deposit and dissemination of scientific research documents, whether they are published or not. The documents may come from teaching and research institutions in France or abroad, or from public or private research centers.
L'archive ouverte pluridisciplinaire HAL, est destinée au dépôt et à la diffusion de documents scientifiques de niveau recherche, publiés ou non, émanant des établissements d'enseignement et de recherche français ou étrangers, des laboratoires publics ou privés. 


\title{
Robust damage assessment of multiple cracks based on the Frequency Response Function and the Constitutive Relation Error updating method
}

\author{
B. Faverjon ${ }^{1}$ and J-J. Sinou ${ }^{2}$ \\ ${ }^{1}$ LMT-Cachan UMR-CNRS 8535 (E.N.S. de Cachan / Université Paris 6 / CNRS ), 61 avenue du Président \\ Wilson, 94235 Cachan Cedex, France. Email: faverjon@1mt.ens-cachan.fr \\ ${ }^{2}$ Laboratoire de Tribologie et Dynamique des Systèmes UMR-CNRS 5513, Equipe Dynamique des \\ Structures et des Systèmes, Ecole Centrale de Lyon, 36 avenue Guy de Collongue, 69134 Ecully Cedex, \\ France. Email: jean-jacques.sinou@ec-lyon.fr
}

\begin{abstract}
The aim of this paper is to present a damage assessment technique for the non destructive detection and sizing of multiple open cracks in beams. The Constitutive Relation Error updating method is used for the identification of the location and the size of multi-cracks in a simply supported beam.

The present identification method is illustrated through numerical examples including double and triples cracks. Moreover, the efficiency and robustness of the proposed method is demonstrated through various numerical simulations in regard to the non-dimensional crack depth and the crack location.

It is demonstrated that the Constitutive Relation Error updating method can detect the number of cracks on the beam and can estimate both the crack positions and sizes with satisfactory precision, even if $10 \%$ or $20 \%$ noise levels has been added to the simulations, and only few degrees of freedom are used for the identification procedure.
\end{abstract}

\section{Introduction}

It is well known that catastrophic mechanical structural failure may be caused by fatigue cracks. In order to avoid failure caused by cracks, many researchers have performed extensive investigations and damage assessment techniques based on vibration measurements. The damage assessment may be divided in a two-phases approach : firstly the damage is located and then then degree of damage is quantified. For example, Friswell et al. [1] used an application of genetic algorithm to locate damage in a beam and in a cantilever plate structure. After, the location of the crack, the crack depth is estimated by using a eigensensitivity method.

The problem of a structure with multiple cracks has received less attention due to the robust damage assessment techniques needed, and only relatively few papers deal with the problem of multiple cracks assessment for structures. Effectively, if the structure has multiple cracks, the identification procedure is more complex due to the fact that the proposed damage assessment technique must estimate not only the crack positions and depths, but also the number of cracks in the structure. As explained by Alvandia and Cremona [2], usual vibration-based damage identification techniques may show less efficiency in the case of complex and simultaneous damages.

The number of cracks present in a structure will usually be unknown for practical engineering applications. So, the development of damage assessment techniques of multiple cracks may be very useful and of important interest. For example, Khiem and Lien [3] used the Dynamic Stiffness Matrix approach in order to detect not 
only the crack position and depth, but also the quantity of possible cracks. But they indicated that the obtained results show that the procedure developed works effectively only for measurement errors not exceeding $7 \%$. Ruotolo and Surace [4] proposed to identify multiple cracks by using the inverse problem as an optimization task solved by means of a genetic algorithm. They validated this damage assessment technique by considering both simulated and experimental data and demonstrated that this method permits assessment of the number of cracks induced on the beam and can estimate the crack positions and depths with satisfactory precision. Kisa and Gurel [5] used damage from changes in the natural frequencies and in the mode shapes of a beam in order to detect an arbitrary number of cracks and the associated crack depths and positions. They concluded that the modal data may provide useful information for multiple cracks detection with a reasonable computational time. However, it may be remained that such methods are less efficiency in the case of damaged structures with measurement errors.

Chang and Chen [6] presented a damage assessment technique based on spatial wavelet analysis. The positions and depths of the cracks are predicted with acceptable precision even though there are many cracks in the beam. However, they indicated that the limitation of the proposed technique is that the crack cannot be detected when the crack location is near the boundaries due to the fact that there are two peaks near the boundaries in the wavelet plot.

In this paper, we propose to use the Frequency Response Function (FRF) and the Constitutive Relation Error updating method (the CRE updating method) that relies on a parametric model of the structure and the minimization of a penalty function based on the error between the experimental data and the predictions from the model. This method belongs to the model updating methods that minimize the discrepancy between the test data and the model by modifying the numerical model. A state-of-the-art review of such updating methods can be found in [7]. The "direct methods" [8,9] perform the corrections of the mass and stiffness matrices of the model but do not unfortunately take into account the physical meaning of the modifications. The "indirect or parametric methods" update models from changes of physical parameters of the model. Three categories of cost functions can be used : the input residuals $[10,11]$, the output residuals $[12,13]$ and the residual named the "Constitutive Relation Error (CRE)" which is used in this paper. It provides a measure of quality of the updated model which is essential for model validation [14-17].

In this study, it will be demonstrated that the proposed assessment technique (the Constitutive Relation Error updating method) may detect not only the number of cracks but also the crack locations and depths, even if large measurement errors (10\% and 20\% noise levels have been added to the simulations) and few sensors may be for the identification procedure.

The paper is organized as follows. Section 2 summarizes the concept of the Constitutive Relation Error updating method. Then, the mechanical system with the transverse open crack under study is discussed in Section 3. The numerical studies showing the application of the proposed damage assessment technique are also presented. Firstly, the identification of the multiple cracks locations ans depths are investigated by considering the Frequency Response Function throughout the beam, in one transverse direction and without noise on measurements. However, as previously explained by Friswell [18], environmental effects may induced changes in the measured data that make damage assessment very difficult. Moreover, one very difficult aspect of damage location is the number of the sensors. Considering these two aspects of damage assessment, noise levels will be added to the numerical simulations, and only few degrees of freedom will be used for the identification procedure. Finally, conclusions are given in Section 4. 


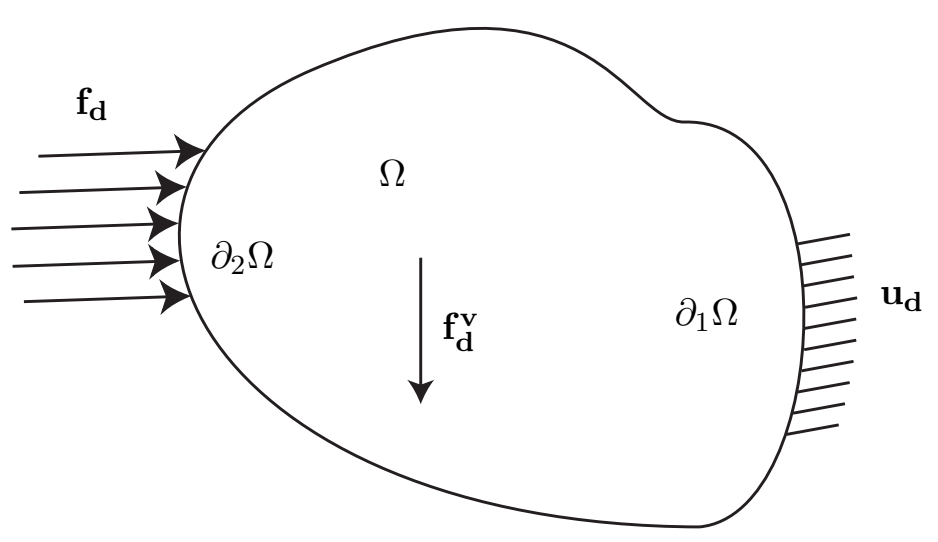

Figure 1: Domain studied and applied loads

\section{The Constitutive Relation error method}

The constitutive relation error updating method is one of methods used for validation in structural dynamics problems. Its concept consists in using an a posteriori estimator and its efficiency has previously been shown [14-17]. The method is based on the Drucker error. In this section, we present the method for the case of a general problem and it will be applied for vibrations of a simply supported beam in Section 3.

\subsection{The construction of the cost function}

Consider a structure of domain $\Omega$ (see Figure 1) vibrating during a time interval $t \in[0, T]$. Displacements $\mathbf{u}_{\mathbf{d}}$ and forces $\mathbf{f}_{\mathbf{d}}$ are prescribed on boundaries $\partial \Omega_{1}$ and $\partial \Omega_{2}$ respectively, besides we have $\partial \Omega_{1} \cup \partial \Omega_{2}=\partial \Omega$. Plus, forces $\mathbf{f}_{\mathbf{d}}^{\mathbf{v}}$ are body forces in domain $\Omega$.

The method consists in finding solution $\mathbf{s}(\mathbf{M}, \mathbf{t})=(\mathbf{u}(\mathbf{M}, \mathbf{t}), \sigma(\mathbf{M}, \mathbf{t}), \boldsymbol{\Gamma}(\mathbf{M}, \mathbf{t})), \mathbf{M} \in \boldsymbol{\Omega}, t \in[0, T]$, which verifies a set of reliable equations and a set of less reliable ones. $\mathbf{M}$ is the position vector, $\mathbf{u}$ the displacement, $\boldsymbol{\sigma}$ the stresses and $\boldsymbol{\Gamma}(\mathbf{M}, \mathbf{t})$ the forces. The reliable equations are the kinematic constraints and the equilibrium equations. The less reliable equations will lead to the construction of the error, on which the minimization will be done. The two constitutive relations are given by

$$
\begin{gathered}
\boldsymbol{\sigma}=(\mathbf{H}+i \omega \mathbf{B}) \boldsymbol{\varepsilon}(\mathbf{u}), \\
\boldsymbol{\Gamma}=-\rho \omega^{\mathbf{2}} \mathbf{u},
\end{gathered}
$$

where $\mathbf{H}$ and $\mathbf{B}$ are the Hooke's and damping operators, $\varepsilon$ the strain tensor and $\rho$ the density. It should be noted that, since we consider in this paper only the case of forced vibrations problems, the equations are then written in the frequency domain; $\omega$ being the measure angular frequency. Besides, the solution is admissible (that verifies the equations considered reliable). In the context of model updating, it is necessary to include data coming from measurements. Again, these types of data are subdivided into two sets of equation : one reliable and one less reliable. If we consider the case of a structure excited in one point on which displacements are measured at different locations, the reliable group consists in $\omega$, the positions and directions of the excitations and sensors whereas the amplitudes of forces $\widetilde{\mathbf{f}}_{\mathbf{d}}$ and displacement $\widetilde{\mathbf{u}}_{\mathbf{d}}$ at the excitations and sensors points are considered less reliable measurements. Then, the total error $e_{\omega}^{2}$ consists in a term devoted to error on modeling $\zeta_{\omega}^{2}$ and another term related to error on measurements $\eta_{\omega}^{2}$. The problem to be solved is to find admissible fields 
$\mathbf{s}$ or equivalently $\mathbf{s}^{\prime}=(\mathbf{u}, \mathbf{v}, \mathbf{w})$ where $\mathbf{u}, \mathbf{v}, \mathbf{w}$ are related to static (subscript $s$ ) and kinematic (subscript $c$ ) quantities by :

$$
\begin{gathered}
\mathbf{u}_{\mathbf{c}}=\mathbf{u}, \\
\boldsymbol{\sigma}_{\mathbf{s}}=(\mathbf{H}+\mathbf{i} \omega \mathbf{B}) \varepsilon(\mathbf{v}), \\
\boldsymbol{\Gamma}_{\mathbf{s}}=-\rho \omega^{2} \mathbf{w},
\end{gathered}
$$

The solution of the problem is obtained by minimizing the modified constitutive relation error $e_{\omega}^{2}$, a cost function relating static and kinematic quantities [16], which is written, using $\mathbf{u}, \mathbf{v}, \mathbf{w}$, such as

$$
e_{\omega}^{2}=\frac{\zeta_{\omega}^{2}}{D_{\omega}^{2}}+\frac{r}{1-r} \eta_{\omega}^{2}
$$

with

$$
\begin{array}{r}
\zeta_{\omega}^{2}(\mathbf{u}, \mathbf{v}, \mathbf{w})=\int_{\mathbf{\Omega}} \frac{\gamma}{\mathbf{2}} \operatorname{tr}\left[\left(\mathbf{H}+\mathbf{T} \omega^{\mathbf{2}} \mathbf{B}\right)\left(\varepsilon(\mathbf{v})-\varepsilon(\mathbf{u})^{\star}(\varepsilon(\mathbf{v})-\varepsilon(\mathbf{u}))\right]\right. \\
+\frac{1-\gamma}{2} \rho \omega^{2}(\mathbf{u}-\mathbf{w})^{\star}(\mathbf{u}-\mathbf{w}) \mathbf{d} \boldsymbol{\Omega}
\end{array}
$$

and

$$
\eta_{\omega}^{2}=\frac{\left\|\left.\mathbf{u}\right|_{\partial_{1} \Omega}-\widetilde{\mathbf{u}}_{\mathbf{d}}\right\|^{2}}{\left\|\widetilde{\mathbf{u}}_{\mathbf{d}}\right\|^{2}}+\frac{\left\|\left.\mathbf{f}\right|_{\partial_{2} \Omega}-\widetilde{\mathbf{f}}_{\mathbf{d}}\right\|^{2}}{\left\|\widetilde{\mathbf{f}}_{\mathbf{d}}\right\|^{2}}
$$

$r$ is a weighting coefficient representing how much we trust the experimental data. Previous works [19] gave $r=0.5$. Symbol ${ }^{\star}$ denotes the complex conjugate. $\mathbf{f}$ is the force vector. Denominator $D_{\omega}^{2}$ and norms used have been chosen to ensure both error terms to have equivalent weights such as

$$
D_{\omega}^{2}=\int_{\Omega}\left(\frac{\gamma}{2} \operatorname{tr}\left[\left(\mathbf{H}+T \omega^{2} \mathbf{B}\right) \varepsilon(\mathbf{u})^{\star} \varepsilon(\mathbf{u})\right]+\frac{\mathbf{1}-\gamma}{\mathbf{2}} \rho \omega^{2} \mathbf{u}^{\star} \mathbf{u}\right) \mathbf{d} \boldsymbol{\Omega} .
$$

Generally, we will use error quantities integrated over frequency range $\left[\omega_{\min }, \omega_{\max }\right]$. They are denoted by a subscript $T$ and are calculated by using a weighting factor $z(\omega)$, verifying $\int_{\omega_{\min }}^{\omega_{\max }} z(\omega) d \omega=1$ with $z(\omega) \geq 0$, e.g. $z(\omega)=1 /\left(\omega_{\max }-\omega_{\min }\right)$. The modified error $e_{T}^{2}$ is then given by

$$
e_{T}^{2}=\zeta_{T}^{2}+\eta_{T}^{2}
$$

in which

$$
\zeta_{T}^{2}=\int_{\omega_{\min }}^{\omega_{\max }} \frac{\zeta_{\omega}^{2}}{D_{\omega}^{2}} z(\omega) d \omega \quad \text { and } \quad \eta_{T}^{2}=\int_{\omega_{\min }}^{\omega_{\max }} \eta_{\omega}^{2} z(\omega) d \omega
$$

\subsection{The updating method}

For each frequency $\omega$, we have to solve the problem described previously. This is done through the computations of $\zeta_{T}^{2}$ and $e_{T}^{2}$. Error in modeling $\zeta_{T}^{2}$ gives the relative quality (in \%) of the numerical model with respect to measurements over a frequency range and permits to decide whether the model updating is necessary. The method consists in two steps. The first one, the localization step, selects the substructures having a modeling error higher than a given value :

$$
\zeta_{E T}^{2} \geq \tilde{\delta} \max _{E \in \mathbf{E}} \zeta_{E T}^{2}
$$


where $\mathbf{E}$ includes all the substructures and $\tilde{\delta}$ is a given number.

The second step is the correction process during which error $e_{T}^{2}$ is minimized by updating the parameters from these substructures alone. Each step of the minimization needs to reassemble mass, stiffness and damping matrices. For solving the non linear problem with respect to the parameters, we use a BFGS-based minimization algorithm and gradients of parameters are calculated numerically. Once the correction has been made, error $\zeta_{T}^{2}$ is calculated again. If its new value is smaller than a given level, then the updating process is finished, if it is not, then a new iteration consisting of a localization step and a correction step is performed.

Let $\mathbf{U}, \mathbf{V}$ and $\mathbf{W}$ be the vectors of the nodal values of the displacements fields $\mathbf{u}, \mathbf{v}$ and $\mathbf{w}$. Without loss of generality, we will consider the case of a single excitation so that the measured displacements are normalized by the amplitude of the force vector and consequently only the amplitudes of the displacements appear in the expression of error on measurements $\eta_{\omega}^{2}$. Then, the discrete form of the modified error is written as

$$
\begin{aligned}
e_{\omega}^{2}(\mathbf{U}, \mathbf{V}, \mathbf{W})=\frac{\gamma}{\mathbf{2}}(\mathbf{U}-\mathbf{V})^{\mathbf{T} \star}\left(\mathbf{K}+\mathbf{T} \omega^{\mathbf{2}} \mathbf{C}\right)(\mathbf{U}-\mathbf{V}) & +\frac{\mathbf{1}-\gamma}{\mathbf{2}} \omega^{\mathbf{2}}(\mathbf{U}-\mathbf{W})^{\mathbf{T} \star} \mathbf{M}(\mathbf{U}-\mathbf{W}) \\
& +\frac{r}{1-r}(\mathbf{\Pi} \mathbf{U}-\tilde{\mathbf{U}})^{\mathbf{T} \star} \mathbf{G}(\mathbf{\Pi} \mathbf{U}-\tilde{\mathbf{U}}) .
\end{aligned}
$$

$\boldsymbol{\Pi}$ is a projection operator which when applied to a vector $\mathbf{Z}$ gives the value of this vector at the sensors. The error in measurements is quantified through matrix G. Previous works [20] showed the efficiency of the following expression

$$
\mathbf{G}=\frac{\gamma}{2}\left(\mathbf{k}+T \omega^{2} \mathbf{c}\right)+\frac{1-\gamma}{2} \omega^{2} \mathbf{m}
$$

where $\mathbf{m}, \mathbf{k}$ and $\mathbf{c}$ are the reduced mass, stiffness and damping matrices of the system at measurement points respectively. Besides, the solution must be admissible that is it must satisfy

$$
(\mathbf{K}+i \omega \mathbf{C}) \mathbf{V}-\omega^{2} \mathbf{M W}=\mathbf{F},
$$

where $\mathbf{F}$ is the excitation force vector. Finally, minimizing error $e_{\omega}^{2}$ is done under the admissibility constraints and is obtained by introducing Lagrange multipliers which yields to the resolution of the following system of linear equations :

$$
\mathbf{A Y}=\mathbf{B}
$$

where $\mathbf{A}, \mathbf{Y}$ and $\mathbf{B}$ are written as

$$
\begin{gathered}
\mathbf{A}=\left[\begin{array}{ccc}
\frac{\gamma}{2}\left(\mathbf{K}+T \omega^{2} \mathbf{C}\right) & \frac{1-\gamma}{2} \omega^{2} \mathbf{M} & \frac{r}{1-r} \mathbf{\Pi}^{\mathbf{T}} \mathbf{G} \boldsymbol{\Pi} \\
\frac{\gamma}{2}\left(\mathbf{K}+T \omega^{2} \mathbf{C}\right) & \frac{1-\gamma}{2}(\mathbf{K}-i \omega \mathbf{C}) & \mathbf{0} \\
-\mathbf{K}-i \omega \mathbf{C} & \omega^{2} \mathbf{M} & \mathbf{K}+i \omega \mathbf{C}-\omega^{2} \mathbf{M}
\end{array}\right] \\
\mathbf{Y}=\left[\begin{array}{c}
\mathbf{U}-\mathbf{V} \\
\mathbf{U}-\mathbf{W} \\
\mathbf{U}
\end{array}\right] \\
\mathbf{B}=\left[\begin{array}{c}
\frac{r}{1-r} \mathbf{\Pi}^{\mathbf{T}} \mathbf{G} \widetilde{\mathbf{U}} \\
\mathbf{0} \\
\mathbf{F}
\end{array}\right] .
\end{gathered}
$$




\begin{tabular}{cc}
\hline & \\
Young modulus $E(\mathrm{~Pa})$ & $2 \times 10^{11}$ \\
Shear modulus $G(\mathrm{~Pa})$ & $7.1 \times 10^{10}$ \\
Density $\left(\mathrm{kg} / \mathrm{m}^{3}\right)$ & 7800 \\
Poisson ratio $\nu$ & 0.3 \\
Length $L(\mathrm{~m})$ & 0.05 \\
Radius of the cross section $R(\mathrm{~m})$ & 1 \\
Damping coefficient $\alpha$ & 0.66 \\
Damping coefficient $\beta$ & $1.2 \times 10^{-6}$ \\
Frequency range $(\mathrm{rad} / \mathrm{s})$ & $25000]$ \\
Discretization of the frequency range $(\mathrm{rad} / \mathrm{s})$ & 20 \\
Location, directions of the excitation & node 5, horizontal and vertical \\
Amplitude $(\mathrm{N})$ of the excitation & $1 / \sqrt{2}$ \\
\hline
\end{tabular}

Table 1: Geometrical and physical parameters for the beam, frequency range of the study and nature of the excitation

\section{Application on the identification of multi-cracks for a simply supported beam}

\subsection{Model of the multi-cracks beam}

The layout of the system with multi-cracks under consideration is shown in Figure 2. The system is composed of a circular beam of radius $R$ and length $L$ simply supported at each end. All values of the physical parameters are given in Table 1.

Using a finite element method, the circular beam is discretized into 30 beam finite elements, with four degrees of freedoms at each node [21]. The axial and torsional degrees of freedom are not considered here. The equations of the uncracked system can be written as

$$
\mathbf{M} \ddot{\mathbf{X}}+\mathbf{C} \dot{\mathbf{X}}+\mathbf{K X}=\mathbf{0}
$$

where $\mathbf{X}$ is the vector of the degrees-of-freedom and dot represents the derivative with respect to the time. Proportional damping matrix $\mathbf{C}$ can be expressed as $\mathbf{C}=\alpha \mathbf{M}+\beta \mathbf{K}$ with $\alpha$ and $\beta$ real constants.

Due to strain energy concentration in the vicinity of the tip of the crack under load, the presence of a transverse crack introduces local flexibility. Mayes and Davies [22,23] proposed a theoretical model of a transverse crack, by reducing the second moment of area of the element at the location of the crack by $\Delta I$ that is given by

$$
\Delta I=I_{0}\left(\frac{\frac{R}{l}\left(1-\nu^{2}\right) F(\mu)}{1+\frac{R}{l}\left(1-\nu^{2}\right) F(\mu)}\right),
$$

where $I_{0}, R, l$, and $\nu$ are the second moments of area, beam radius, length of the section and Poisson's ratio, respectively. $\mu$ is the non-dimensional crack depth and is given by

$$
\mu=\frac{h}{R}
$$

where $h$ defines the crack depth of the beam, as shown in Figure 2. $F(\mu)$ defines the non-linear compliance as a function of variations in non-dimensional crack depth $\mu$, which can be derived from a series of experiments 


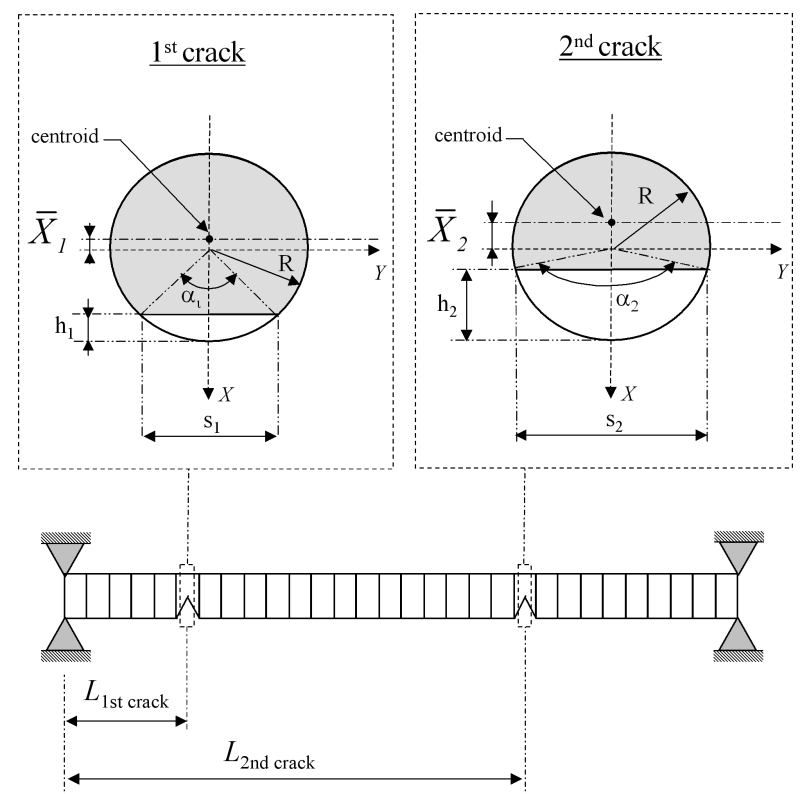

Figure 2: Model of the simply supported beam with multi-cracks

using chordal cracks (see Mayes and Davies [22,23]). At the location of the $i$ th transverse crack, the stiffness matrix $\left[\mathbf{K}_{\text {crack }}^{i}\right]$ is defined (with respect to the principal axes of the crack front) as

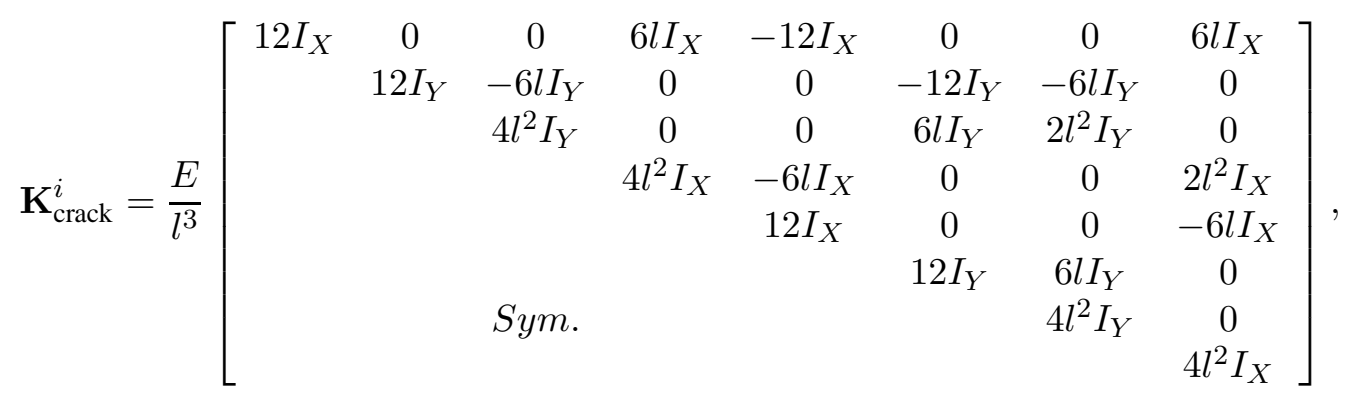

where $I_{X}$ and $I_{Y}$ are the new moments of inertia, about the parallel centroidal axes, due to the presence of the cracked elements. They are given by [24]

$$
\begin{gathered}
I_{X}=\frac{R^{4}}{4}\left((1-\mu)\left(1-4 \mu+2 \mu^{2}\right) \gamma+\frac{\alpha}{2}\right), \\
I_{Y}=\frac{\pi R^{4}}{4}+R^{4}\left(\frac{2}{3}(1-\mu) \gamma^{3}+\frac{1}{4}(1-\mu)\left(1-4 \mu+2 \mu^{2}\right) \gamma+\sin ^{-1}(\gamma)\right)-A \bar{X}^{2},
\end{gathered}
$$

where $A$ and $\bar{X}^{2}$ define the uncracked area of the cross-section and the distance from the axis $X$ to the centroid of the cross section

$$
\begin{gathered}
A=R^{2}\left((1-\mu) \gamma+\frac{\alpha}{2}\right), \\
\bar{X}=\frac{2}{3 A} R^{3} \gamma^{3}
\end{gathered}
$$


where $\gamma$ is equal to $\sqrt{2 \mu-\mu^{2}}$ for convenience. As illustrated in Figure 2, $\alpha$ defines the crack angle and is given by $\alpha=2 \cos ^{-1}(1-\mu)$.

For a transverse beam with double-cracks, the global stiffness matrix $\left[\mathbf{K}_{\text {crack }}\right]$ due to the presence of the cracks is given by

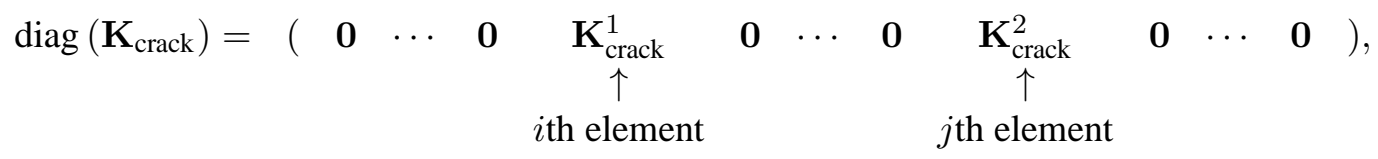

where $\mathbf{K}_{\text {crack }}^{1}$ and $\mathbf{K}_{\text {crack }}^{2}$ are the stiffness matrices that are associated with the first and second cracks, respectively (as indicated in Equation 23). They are located at the $i$ th and $j$ th beam location. $\mathbf{0}$ defines the $8 \times 8$ null matrix.

Finally, the equation of motion for the simply supported beam with multiple cracks and excited by an external force vector $\mathbf{F}_{\mathbf{e}}(\mathbf{t})=\mathbf{F} \mathbf{e}^{\mathbf{i} \omega \mathbf{t}}$ (where $\mathbf{F}$ defines the force amplitude) can be written as

$$
\mathbf{M} \ddot{\mathbf{X}}+\hat{\mathbf{C}} \dot{\mathbf{X}}+\hat{\mathbf{K}} \mathbf{X}=\mathbf{F}_{\mathbf{e}}(\mathbf{t})
$$

with

$$
\begin{aligned}
\hat{\mathbf{K}} & =\mathbf{K}-\mathbf{K}_{\text {crack }}, \\
\hat{\mathbf{C}} & =\alpha \mathbf{M}+\beta \hat{\mathbf{K}} .
\end{aligned}
$$

As indicated in Equation 31, we assume that the presence of cracks affect the damping matrix via the stiffness matrix.

The response vector may be assumed as $\mathbf{X}(\mathbf{t})=\mathbf{X}_{\mathbf{0}} \mathrm{e}^{\mathrm{i} \omega \mathrm{t}}$. Considering Equation 29, the system governing the equation in the frequency domain is given by

$$
\left(-\omega^{2} \mathbf{M}+i \omega \hat{\mathbf{C}}+\hat{\mathbf{K}}\right) \mathbf{X}_{\mathbf{0}}=\mathbf{F} .
$$

So, the identification of each crack location can be undertaken by considering this last relation and the use of the Constitutive Relation Error (CRE) estimator. Equation 15 particularised for the case of the beam uses stress and damping matrices $\hat{\mathbf{K}}$ and $\hat{\mathbf{C}}$ instead of $\mathbf{K}$ and $\mathbf{C}$. Moreover, vector $\mathbf{X}_{\mathbf{0}}$ in Equation 32 will be either $\mathbf{V}$ (stress and damping terms) or $\mathbf{W}$ (mass term) of Equation 15.

Secondly, the calculation of the depth for the $i$ th crack may be obtained by the minimization of the crack depth error $e_{\mu}^{2}$ (with respect to the non-dimensional crack depth $\mu$ )

$$
e_{\mu}^{2}=\sum_{k=1}^{8} \sum_{l=1}^{8}\left(\mathbf{K}_{\text {crack }, k l}^{i}-\mathbf{K}_{\text {ident }, k l}\right)^{2}
$$

where $\mathbf{K}_{\mathrm{crack}, k l}^{i}$ and $\mathbf{K}_{\mathrm{ident}, k l}$ define the theoretical and identified flexibility coefficients, respectively.

\subsection{Numerical results}

In this paper, we chose to present the identification of different damage locations and crack sizes into the beam described in Section 3 and whose properties are given in Table 1. Six cases were considered : cases 1 to 5 correspond to double cracks and case 6 to triple cracks. Details are presented in Table 2. It is known that the identification depends on the informations available like the number, the location and the direction of the sensors, the presence of noise on measurements. Several studies will show the robustness of the method presented 
in this paper.

Firstly, we consider the identification of the cracks for each case, the measurement information is given by sensors throughout the beam and in one transverse direction, no noise is added. If the level of the model error is above than a certain value then the updating method consists in a first step which is the localization step.

Figures 3(a) to 8(a) present local errors at each localization step before each iteration of the updating process, for the six cases. Clearly, each localization gives the element which has the highest local error and which corresponds to the location of the crack. After the first localization, a minimization of the modified error is made by changing only the parameters of the found element. It corresponds to the first iteration shown in the figures. If the level of the model error is still higher than a sufficient value (leading tor the convergence of the method), another iteration is performed (localization plus minimization steps). Since we have multi-cracks, another iteration is needed. The second one gives the element this time corresponding to the other crack of the two existing for cases 1 to 5 (see Figures 3 to 7) and a crack between the two others not yet found for case 6 . Third crack is obtained thanks to the third iteration (see Figure 8). The computation stops when the value of the model error is enough small. At each iteration, local errors are normalized by the maximum local error. Table 3 explains the updating process and gives model and modified errors before the first iteration and at the end of the process. It clearly appears that the identifications of the multi-cracks locations are successively realized for the six cases. Then, the crack depth identification is obtained by considering the minimization of the crack depth error $e_{\mu}^{2}$ as indicated in Equation 33. The identified crack depths for the six cases are resumed in Table 4. Perfect estimations of the crack depths are obtained in all cases.

Generally speaking, the identification of the cracks depends on the value of the normalized crack depth $\mu$ and on the crack location. Effectively, a crack with a high value of the depth will be classically easier to find (for a given location of the crack). Moreover, the cracks will be more difficult to be detected if they are located very close to nodes for the first eigenmodes (for a given normalized crack depth $\mu$ ).

For example, we can see on Figure 3 (case 1) that the first detected crack is the one which is located in the tenth element (near one node of the third mode) and the second one corresponds to the crack located in the fifteenth element (near one node of the second mode). In this case, this is globally due to the fact that the primary identified crack corresponds to the crack with the greater normalized depth $\mu$ : the crack depth $\mu_{1}$ of the first crack (that is equal to 0.8) is greater than the crack depth $\mu_{2}$ of the second crack (that is equal to 0.5). Similar observations can be made for the cases 2, 4 and 5 which correspond to double-cracks identification. Moreover, the same conclusions can be done for the triple-cracks detection, as indicated in Figure 8(a). The three successive identifications of the cracks locations begin with the greater normalized crack depth and finish by the smaller normalized crack depth.

Finally, it may be observed that the first localization step can indicate the locations of the two cracks if the effects of each crack are similar, as indicated in Figures 5 (corresponding to case 3 with two cracks depths equal to 0.5). For cases 2 and 6 (see Figure 3 and 8), all the cracks locations are visible at the first localization step even if the local errors are predominant for the beam element corresponding to the greater depth crack.

Experiments are frequently perturbed by noise measurement. To represent correctly this reality, we added some uniform random noise on the previous deterministic computational experiments. The noise is uniformly distributed in space and over the frequency range. Moreover, only few sensors are generally used to measure the Frequency Response Functions of the multi-cracks systems. Consequently, in order to demonstrate the efficiency and robustness of the proposed method, we computed again cases 1 and 6 by considering experimental data that were carried out through five one-directional displacements alone (instead of all the one-directional displacements along the beam) and on which several noise levels were added : $0 \%, 10 \%$ and $20 \%$.

Table 5 presents the results of the multi-cracks locations obtained for these cases : model and modified errors before the updating process and after the last iteration. Figures 9(a), 9(c) and 9(e) present the identifications of 


\begin{tabular}{ccccccc}
\hline & & & & & \\
Case & Crack location 1 & $\mu_{1}$ & Crack location 2 & $\mu_{2}$ & Crack location 3 & $\mu_{3}$ \\
\hline 1 & 10 & 0.8 & 15 & 0.5 & \\
2 & 10 & 0.1 & 15 & 0.3 & \\
3 & 10 & 0.5 & 15 & 0.5 & \\
4 & 4 & 0.7 & 23 & 0.4 & & \\
5 & 9 & 0.5 & 7 & 0.6 & 25 & 0.7 \\
6 & 10 & 0.8 & 15 & 0.6 & 25 \\
\hline
\end{tabular}

Table 2: Crack damage locations and depths for cases 1 to 6

a

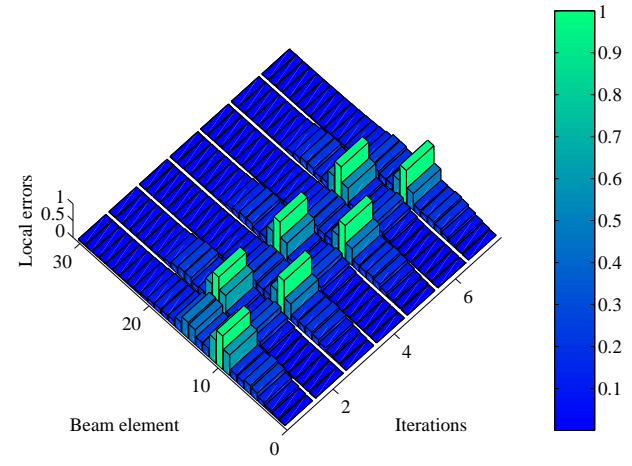

b

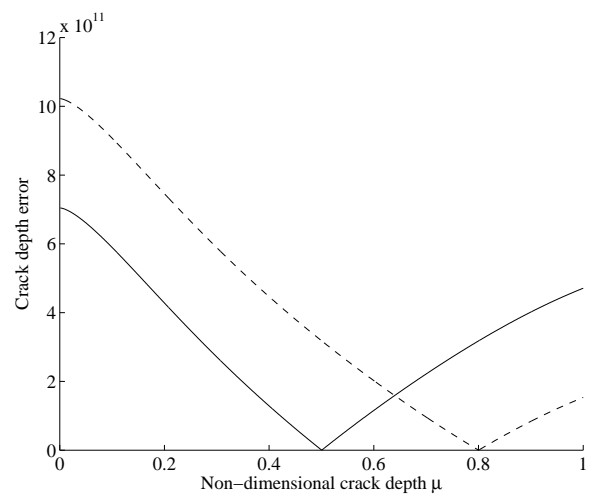

Figure 3: Local errors of the 30 elements, normalized to the largest value, and evolution of the crack depth error for case 1 without noise on measurements (a) Local errors (b) Crack depth error $\left(--\mu_{1},-\mu_{2}\right)$

a

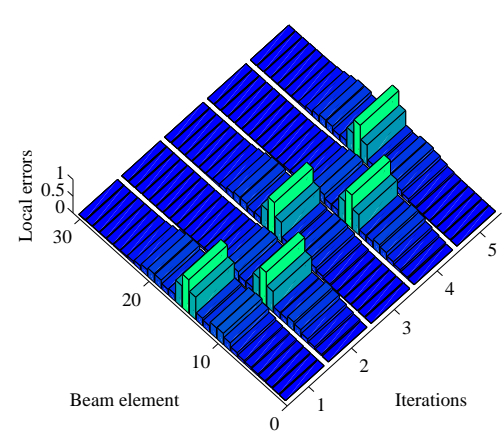

$\mathrm{b}$

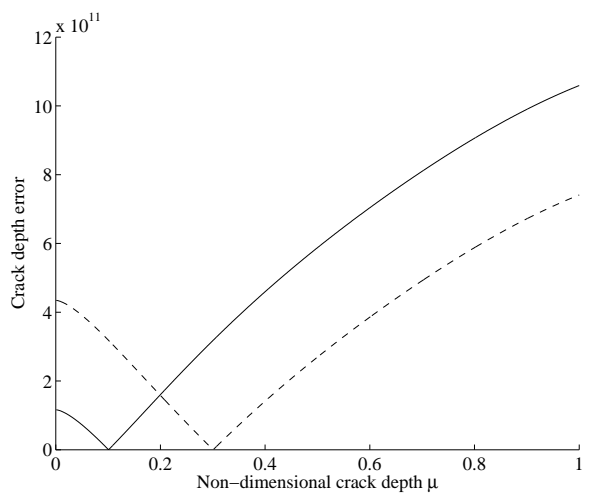

Figure 4: Local errors of the 30 elements, normalized to the largest value, and evolution of the crack depth error for case 2 without noise on measurements (a) Local errors (b) Crack depth error $\left(-\mu_{1},--\mu_{2}\right)$ 
a

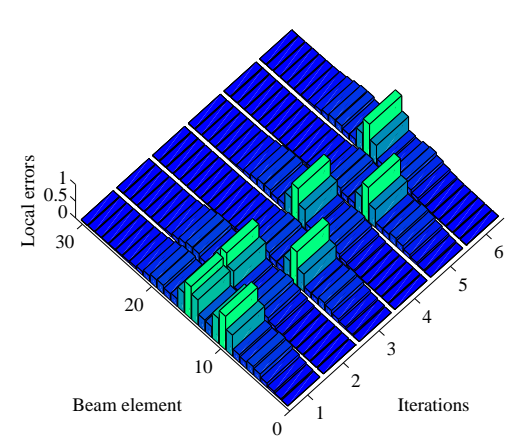

b

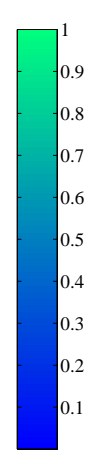

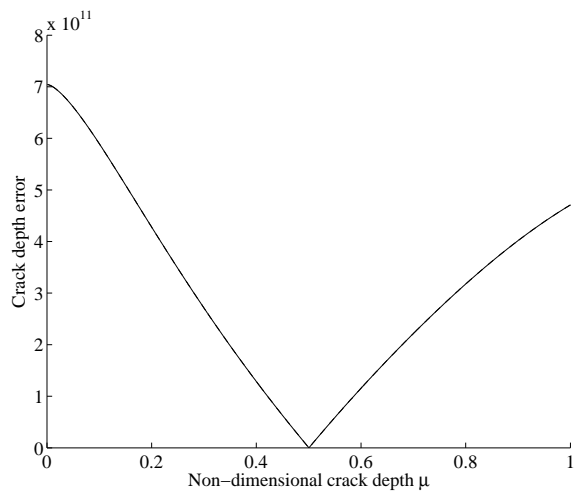

Figure 5: Local errors of the 30 elements, normalized to the largest value, and evolution of the crack depth error for case 3 without noise on measurements (a) Local errors (b) Crack depth error $\left(-\mu_{1},--\mu_{2}\right)$

a

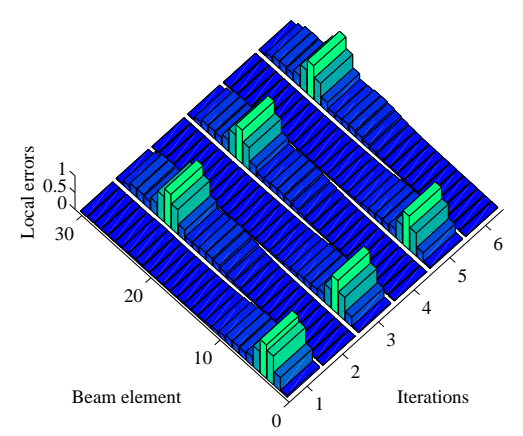

$\mathrm{b}$

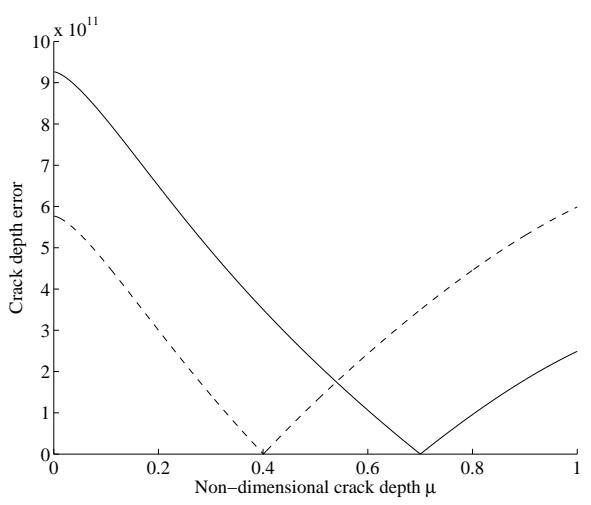

Figure 6: Local errors of the 30 elements, normalized to the largest value, and evolution of the crack depth error for case 4 without noise on measurements (a) Local errors (b) Crack depth error $\left(-\mu_{1},--\mu_{2}\right)$

\begin{tabular}{ccccccc} 
case & noise $(\%)$ & $\zeta_{T}^{2}$ & $e_{T}^{{ }^{i}}$ & $\zeta_{T}^{2}$ & $e_{T}^{{ }^{f}}$ & iterations number \\
\hline 1 & 0 & 2.63 & 3.71 & $1.06 \times 10^{-6}$ & $1.51 \times 10^{-6}$ & 7 \\
2 & 0 & 0.40 & 0.59 & $1.65 \times 10^{-6}$ & $2.48 \times 10^{-6}$ & 5 \\
3 & 0 & 1.36 & 1.91 & $2.84 \times 10^{-6}$ & $4.22 \times 10^{-6}$ & 6 \\
4 & 0 & 2.16 & 3.03 & $0.97 \times 10^{-6}$ & $1.30 \times 10^{-6}$ & 6 \\
5 & 0 & 1.60 & 2.27 & $4.99 \times 10^{-6}$ & $7.08 \times 10^{-6}$ & 8 \\
6 & 0 & 3.12 & 4.46 & $4.28 \times 10^{-6}$ & $5.96 \times 10^{-6}$ & 11 \\
\hline
\end{tabular}

Table 3: Errors (in \%) before the updating process (subscript $i$ ) and after the last iteration needed for convergence of the solution (subscript $f$ ) of the updating process for cases 1 to 6 and measurements without noise 
a

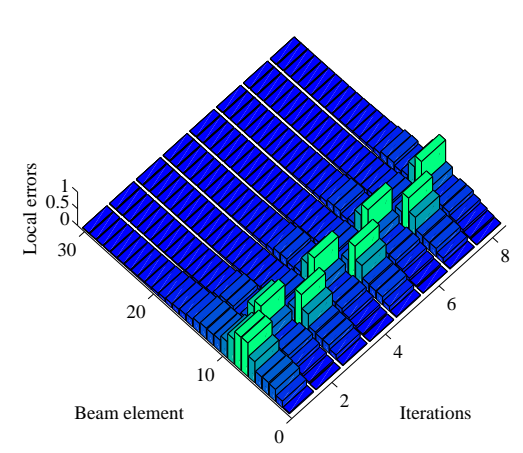

b

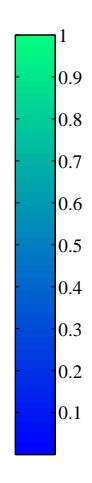

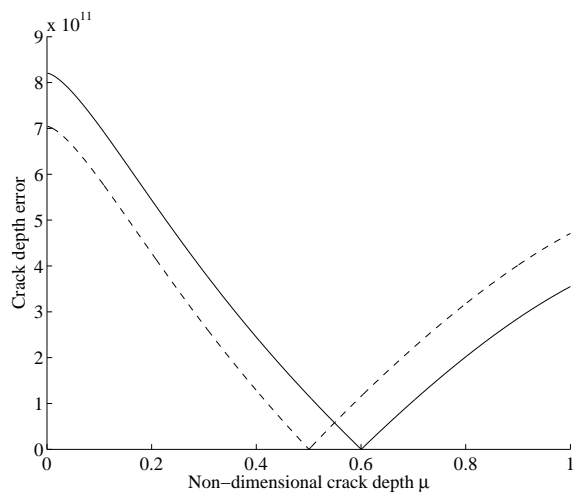

Figure 7: Local errors of the 30 elements, normalized to the largest value, and evolution of the crack depth error for case 5 without noise on measurements (a) Local errors (b) Crack depth error $\left(--\mu_{1},-\mu_{2}\right)$

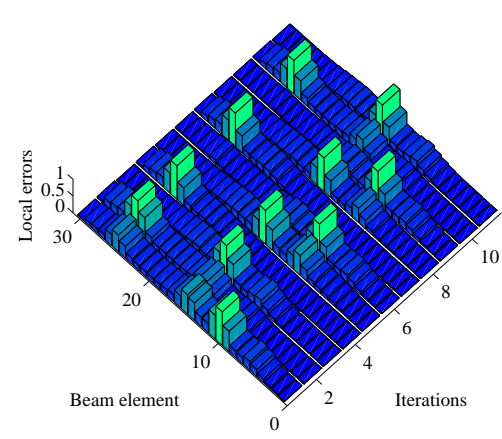

$\mathrm{b}$

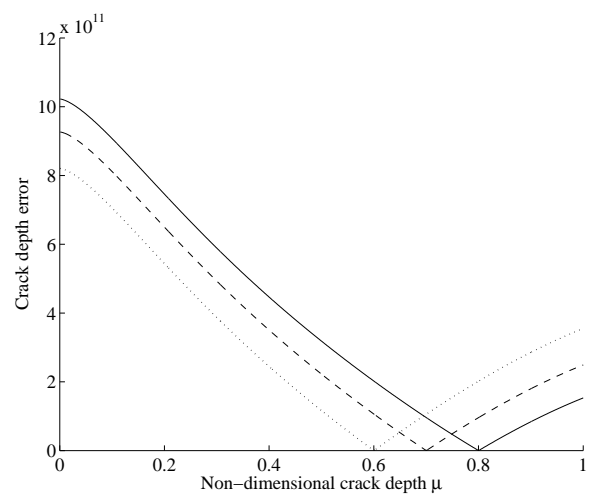

Figure 8: Local errors of the 30 elements, normalized to the largest value, and evolution of the crack depth error for case 6 without noise on measurements (a) Local errors (b) Crack depth error $\left(-\mu_{1}, \cdots \mu_{2},--\mu_{3}\right)$

\begin{tabular}{ccccccc} 
case & assumed $\mu_{1}$ & identified $\mu_{1}$ & assumed $\mu_{2}$ & identified $\mu_{2}$ & assumed $\mu_{3}$ & identified $\mu_{3}$ \\
\hline 1 & 0.800 & 0.800 & 0.500 & 0.500 & & \\
2 & 0.100 & 0.101 & 0.300 & 0.301 & & \\
3 & 0.500 & 0.500 & 0.500 & 0.500 & & \\
4 & 0.700 & 0.700 & 0.400 & 0.401 & & \\
5 & 0.500 & 0.501 & 0.600 & 0.600 & & \\
6 & 0.800 & 0.800 & 0.600 & 0.600 & 0.700 & 0.700 \\
\hline
\end{tabular}

Table 4: Comparison of the assumed and identified non-dimensional crack depths $\mu$ for cases 1 to 6 without noise 


\begin{tabular}{|c|c|c|c|c|c|c|c|}
\hline case & noise $(\%)$ & $\zeta_{T}^{2^{i}}$ & $e_{T}^{2^{i}}$ & $\zeta_{T}^{2 f}$ & $e_{T}^{2 f}$ & & terations number \\
\hline 1 & 0 & 2.57 & 3.05 & $2.65 \times 10^{-6}$ & $3.40 \times 10^{-6}$ & & 8 \\
\hline 1 & 0 & & & $3.41 \times 10^{-4}$ & $3.98 \times 10^{-4}$ & 5 & (intermediate step) \\
\hline 1 & 10 & 2.77 & 3.32 & 0.51 & 0.91 & & 5 \\
\hline 1 & 20 & 3.05 & 3.78 & 0.99 & 1.74 & & 5 \\
\hline 6 & 0 & 2.78 & 3.2 & $2.51 \times 10^{-5}$ & $3.68 \times 10^{-6}$ & & 14 \\
\hline 6 & 0 & & & 0.0072 & 0.0082 & 7 & (intermediate step) \\
\hline 6 & 0 & & & 0.0019 & 0.0022 & 9 & (intermediate step) \\
\hline 6 & 10 & 2.98 & 3.47 & 0.49 & 0.88 & & 9 \\
\hline 6 & 20 & 3.25 & 3.90 & 1.06 & 1.77 & & 7 \\
\hline
\end{tabular}

Table 5: Errors (in \%) before (subscript $i$ ) the updating process and after the last iteration needed for convergence of the solution (subscript $f$ ), for cases 1 and 6 , with sensors located on 5 displacements : nodes 5,10 , 15, 20 and 25, and with various noise levels

the cracks locations with the associated local errors throughout the beam and at each iteration of the updating process for case 1. The same results are shown in Figures 10(a), 10(c) and 10(e) for case 6. Even if only few sensors are available and noise measurement exists, it clearly appears that the final identifications of the multicracks locations are again in perfect agreement with the assumed positions of the two and three cracks for cases 1 and 6 , respectively. Then, the determination of the crack depth $\mu$ of each crack is obtained by minimizing the crack depth error function of Equation 33. Results of the estimated crack depths are given in Table 6. Even if the difference between the estimated and identified non-dimensional crack depths tend to increase by increasing the noise levels, it may be concluded that the results are reasonably good. So it is demonstrated that the damage detection of the multi cracks and the identification of the cracks size and position can be obtained with satisfactory precision, even if $20 \%$ noise level has been added to the simulations, and less than $5 \%$ of the degrees of freedom are measured.

Finally, the update model is shown through the Frequency Response Functions (red dotted-dashed lines) plotted with the angular frequency in Figures 11 to 13, for case 1, and for 0\%, $10 \%$ and 20\% noise levels respectively. These Frequency Response Functions are compared to the assumed Frequency Response Functions (solid line). Moreover, the Frequency Response Functions of the uncracked system are presented (dashed lines). It is clearly observed that these FRFs are far from the FRFs of the system with multi-cracks.

Considering the results presented in Figures 11 to 13, the update model agrees very well with the assumed FRFs of the system with multi-cracks for all these cases. However, we can notice that the discrepancy between the estimated and assumed Frequency Response Functions increases with the noise on measurements. Similarly, results on case 6 are available in Figures 14 to 16. The influence of the noise can also be seen on local errors (Figures 9-10) which are smoothed with the noise level increasing and on the value of errors before and after the updating process (as presented in Table 5) which are higher compared to that from test data without noise. However, whatever the noise levels presented here, the identification is done again successfully, which demonstrates the robustness of the method. 
a

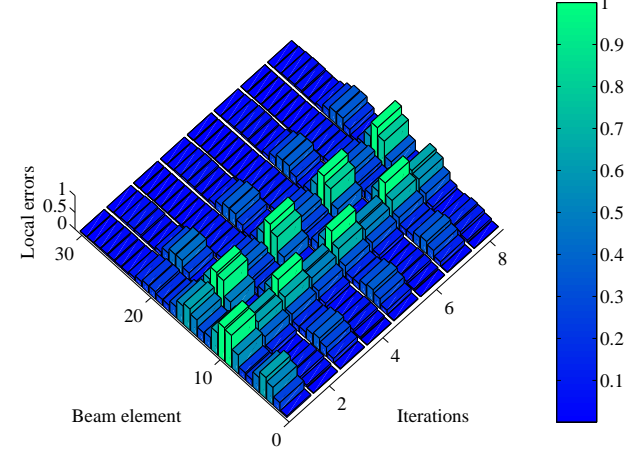

c

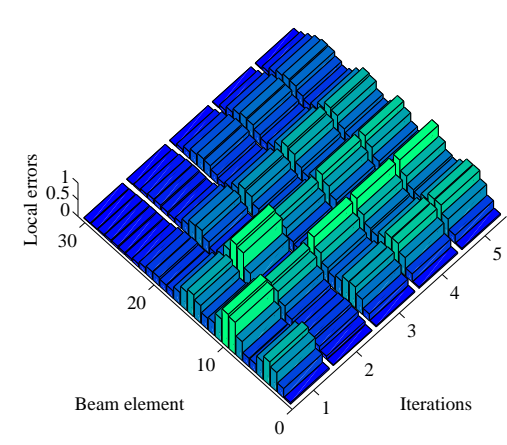

e

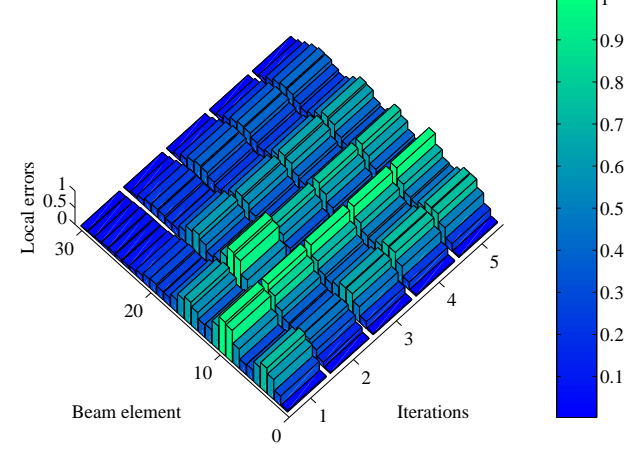

b

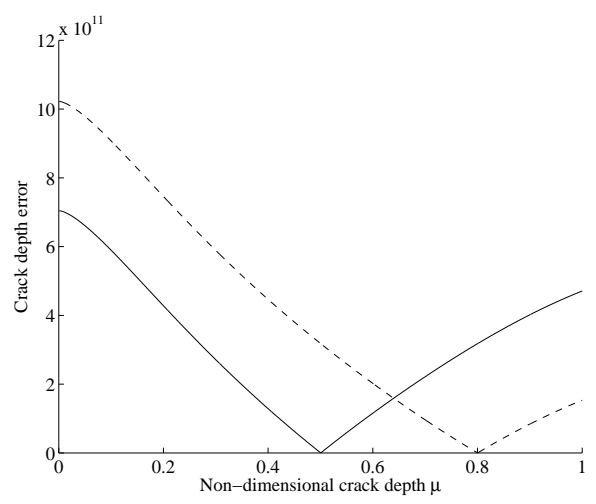

d

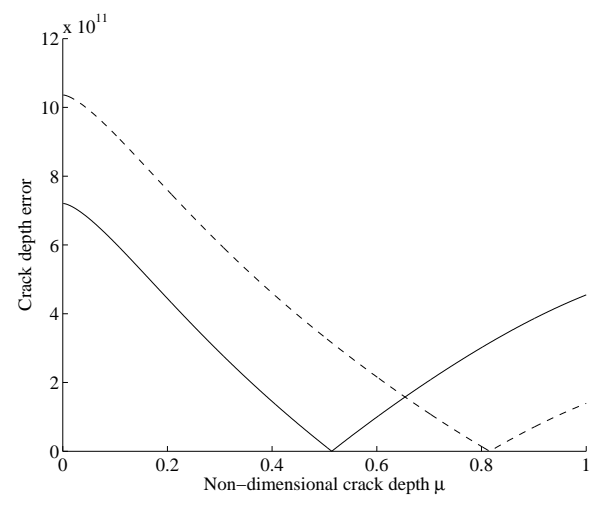

f

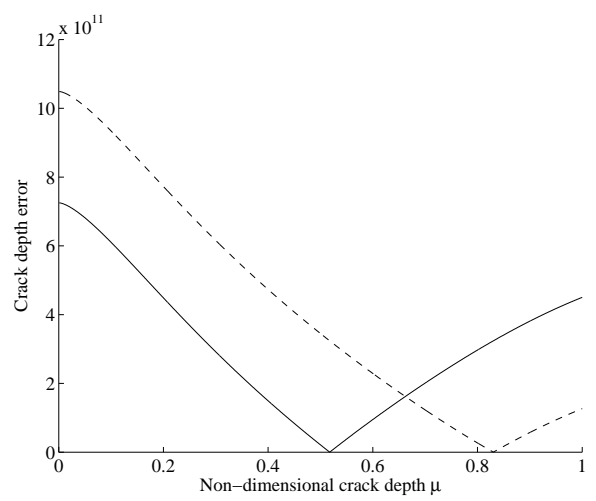

Figure 9: Local errors of the 30 elements, normalized to the largest value, and evolution of the crack depth error for case 1 with noisy measurements (a,c,e) Local errors with $0 \%, 10 \%$ and $20 \%$ of noise (b,d,f) Crack depth error with $0 \%, 10 \%$ and $20 \%$ of noise $\left(--\mu_{1},-\mu_{2}\right)$ 
a

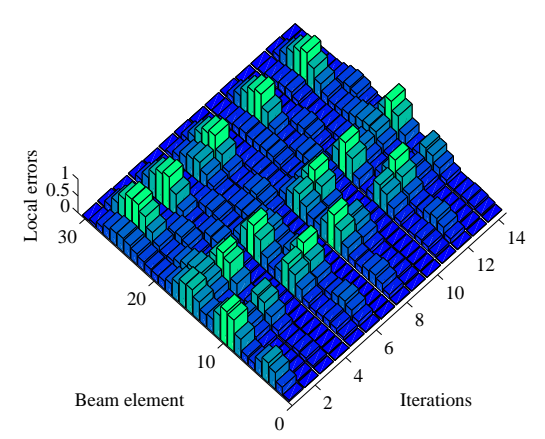

$\mathrm{c}$

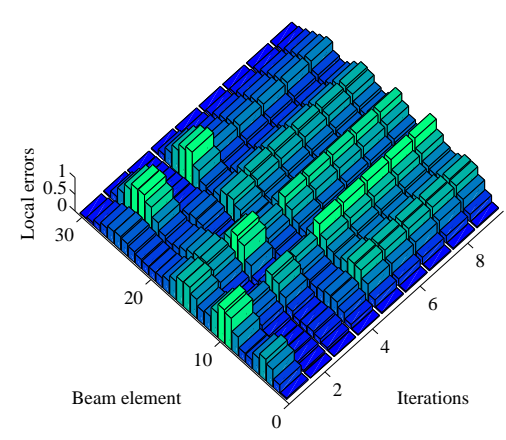

e

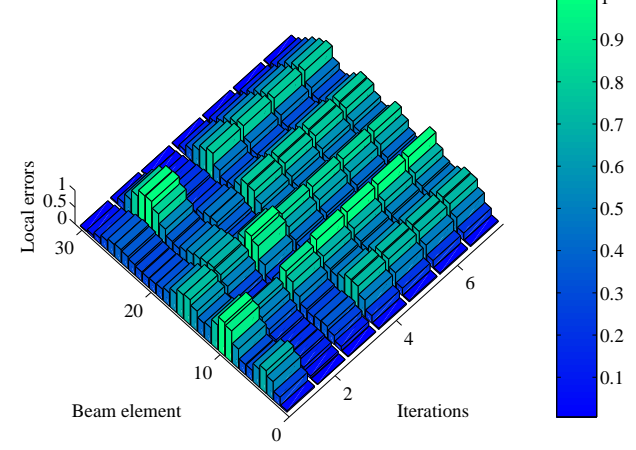

b

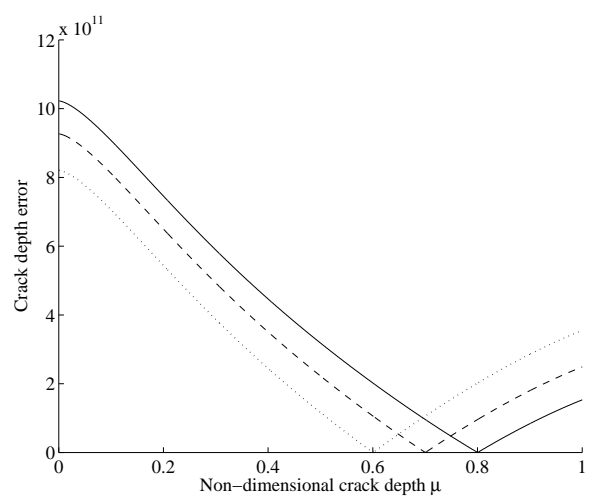

d

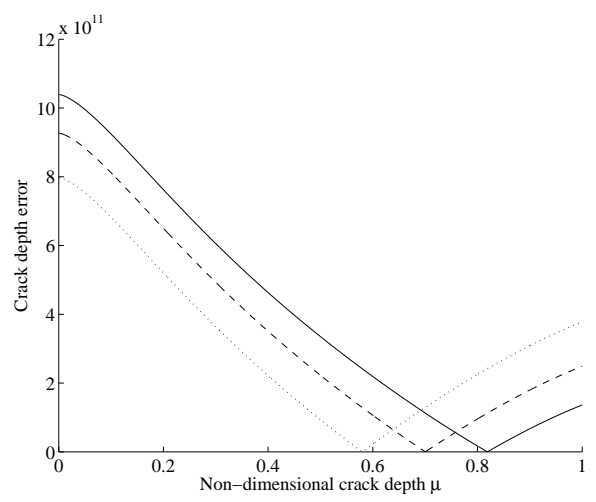

f

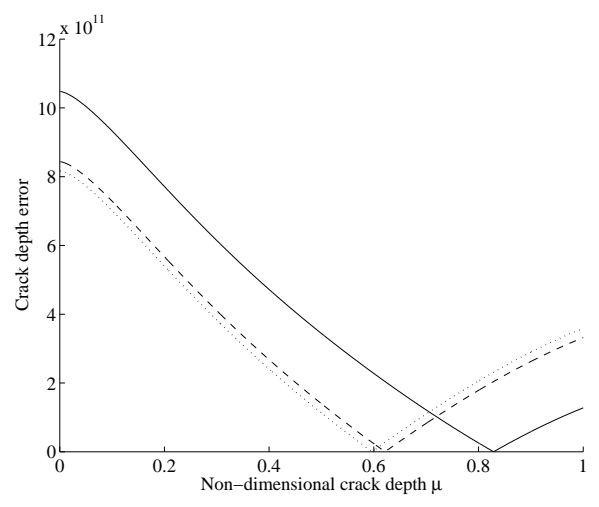

Figure 10: Local errors of the 30 elements, normalized to the largest value, and evolution of the crack depth error for case 6 with noisy measurements (a,c,e) Local errors with $0 \%, 10 \%$ and $20 \%$ of noise (b,d,f) Crack depth error with $0 \%, 10 \%$ and $20 \%$ of noise $\left(-\mu_{1}, \cdots \mu_{2},--\mu_{3}\right)$ 


\begin{tabular}{cccccccc}
\hline & & & & & & \\
case & noise $(\%)$ & assumed $\mu_{1}$ & identified $\mu_{1}$ & assumed $\mu_{2}$ & identified $\mu_{2}$ & assumed $\mu_{3}$ & identified $\mu_{3}$ \\
\hline 1 & 0 & 0.800 & 0.800 & 0.500 & 0.500 & & \\
1 & 10 & 0.800 & 0.816 & 0.500 & 0.514 & & \\
1 & 20 & 0.800 & 0.830 & 0.500 & 0.518 & & \\
6 & 0 & 0.800 & 0.800 & 0.600 & 0.600 & 0.700 & 0.700 \\
6 & 10 & 0.800 & 0.820 & 0.600 & 0.580 & 0.700 & 0.700 \\
6 & 20 & 0.800 & 0.829 & 0.600 & 0.597 & 0.700 & 0.621 \\
\hline
\end{tabular}

Table 6: Comparison of the assumed and identified non-dimensional crack depths $\mu$ for cases 1 and 6 , with sensors located on 5 displacements : nodes $5,10,15,20$ and 25 , and with various noise levels

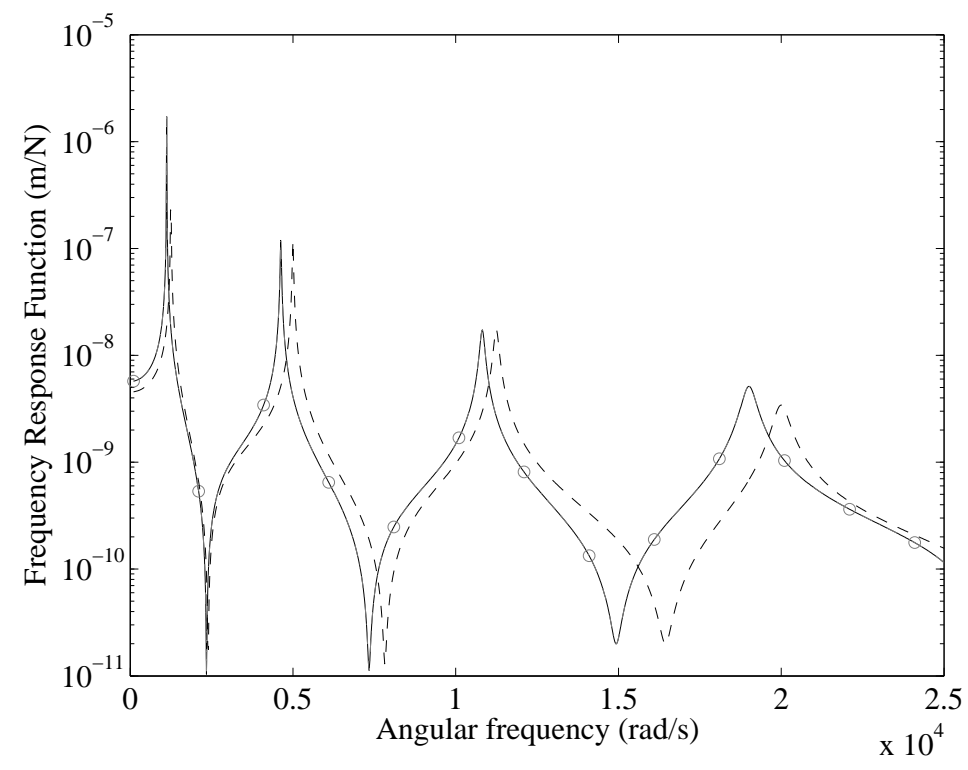

Figure 11: Comparison between the Frequency Response Functions (vertical direction) of the cracked model (solid line), the initial model (dashed lines) and the updated model (dotted-dashed lines with circle symbols), obtained at the beam's element position 5, for case 1, obtained from a computation without random noise and five sensors 


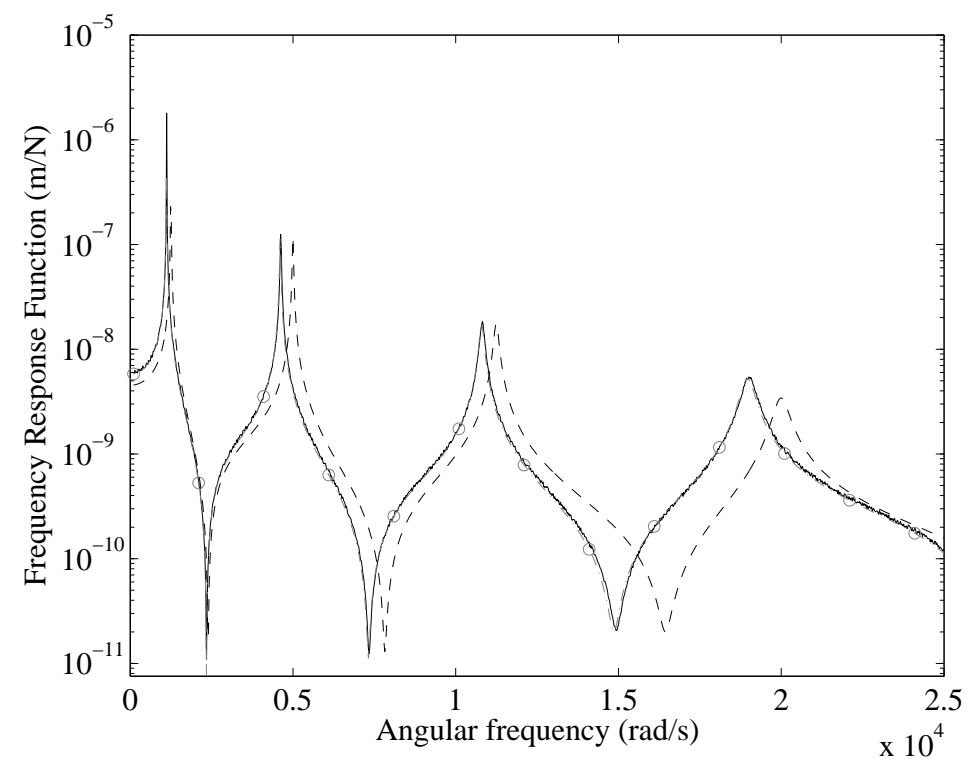

Figure 12: Comparison between the Frequency Response Functions (vertical direction) of the cracked model (solid line), the initial model (dashed lines) and the updated model (dotted-dashed lines with circle symbols), obtained at the beam's element position 5 , for case 1, obtained from a computation with $10 \%$ of random noise and five sensors

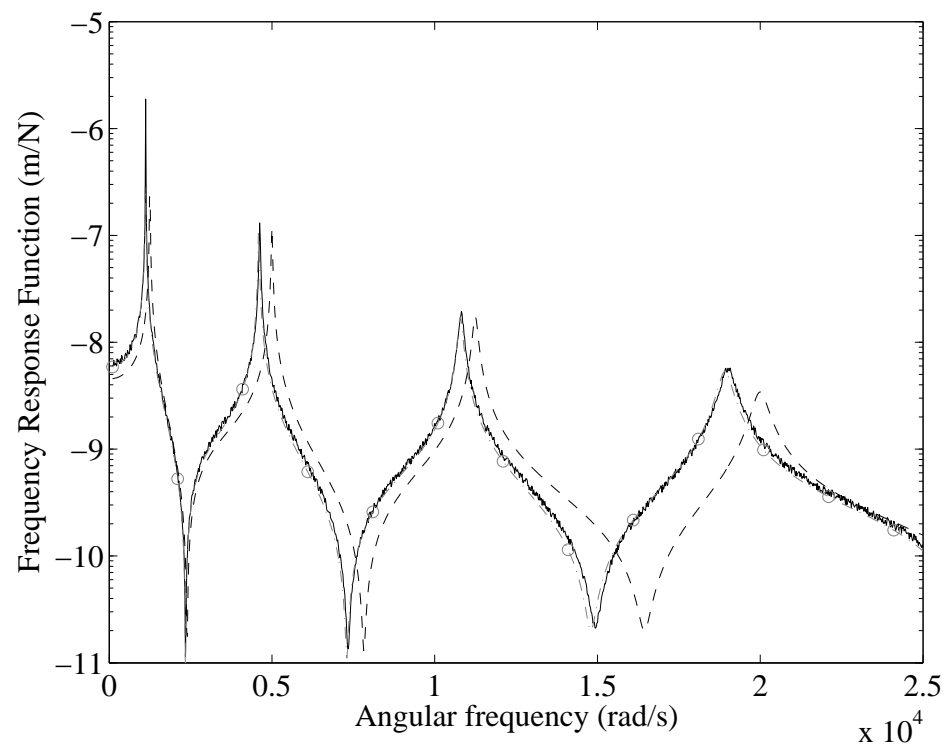

Figure 13: Comparison between the Frequency Response Functions (vertical direction) of the cracked model (solid line), the initial model (dashed lines) and the updated model (dotted-dashed lines with circle symbols), obtained at the beam's element position 5 , for case 1, obtained from a computation with $20 \%$ of random noise and five sensors 


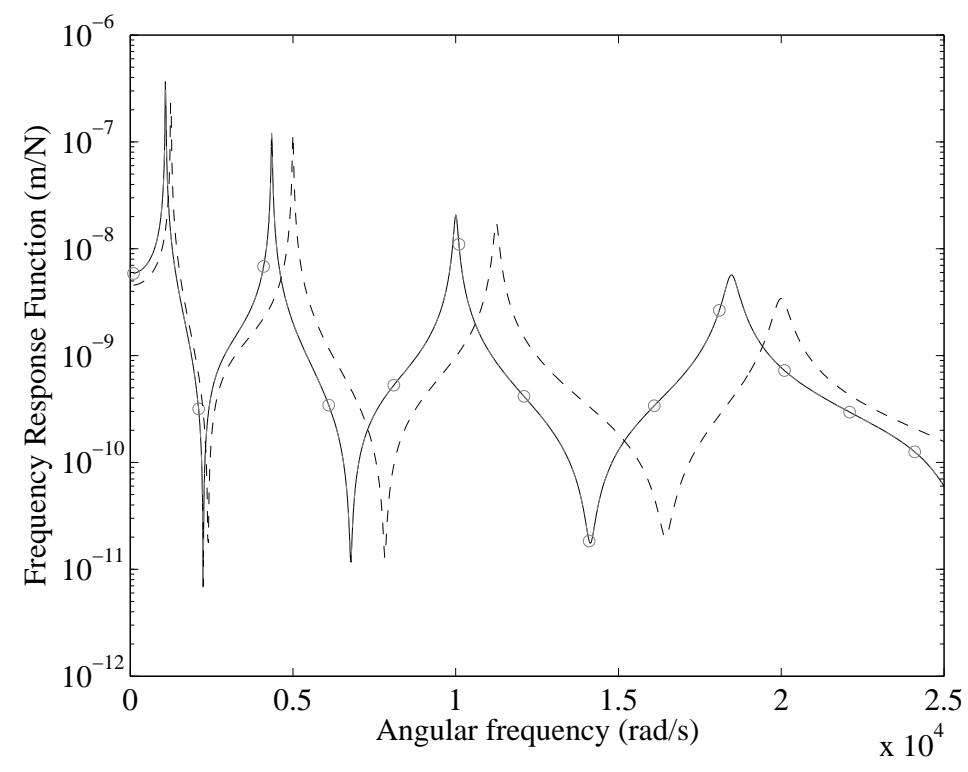

Figure 14: Comparison between the Frequency Response Functions (vertical direction) of the cracked model (solid line), the initial model (dashed lines) and the updated model (dotted-dashed lines with circle symbols), obtained at the beam's element position 5 , for case 6 , obtained from a computation without random noise and five sensors

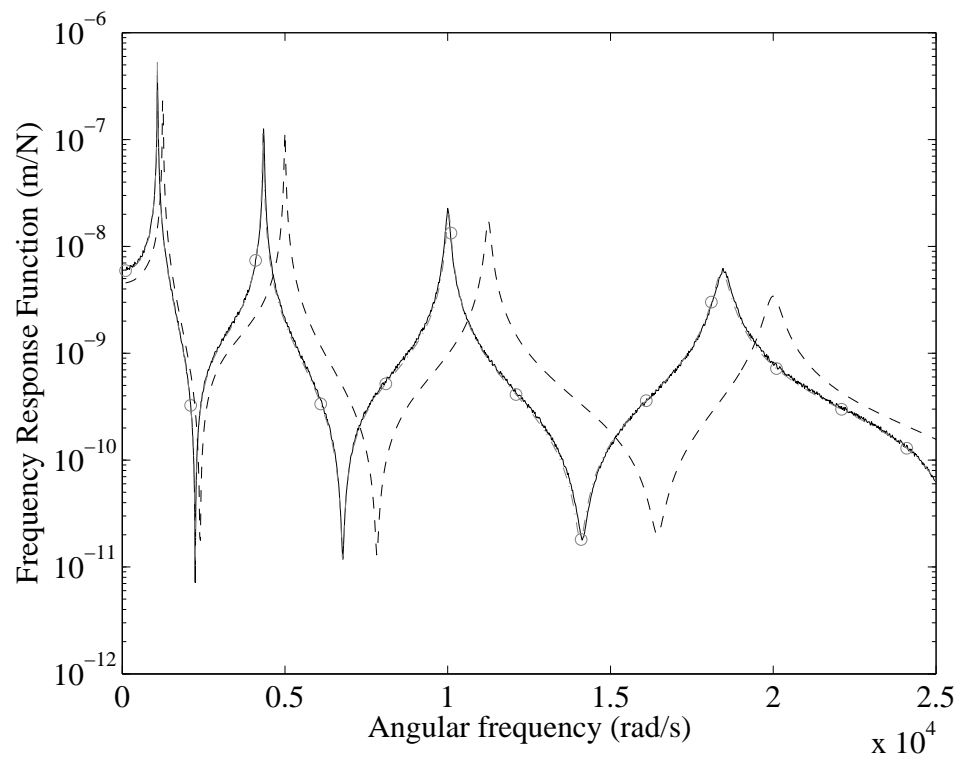

Figure 15: Comparison between the Frequency Response Functions (vertical direction) of the cracked model (solid line), the initial model (dashed lines) and the updated model (dotted-dashed lines with circle symbols), obtained at the beam's element position 5 , for case 6 , obtained from a computation with $10 \%$ of random noise and five sensors 


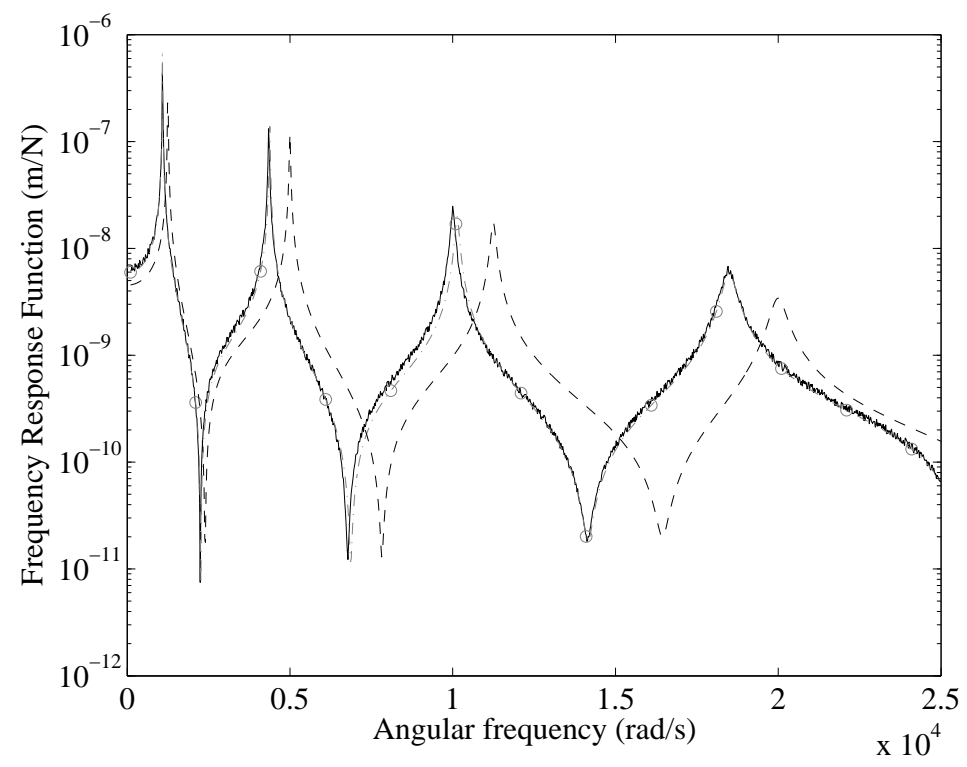

Figure 16: Comparison between the Frequency Response Functions (vertical direction) of the cracked model (solid line), the initial model (dashed lines) and the updated model (dotted-dashed lines with circle symbols), obtained at the beam's element position 5 , for case 6 , obtained from a computation with $20 \%$ of random noise and five sensors

\section{Conclusion}

A non destructive detection of double and triple open transverse cracks for a simply supported beam is proposed in this paper. The damage assessment technique is based on the Constitutive Relation Error updating method and a crack depth error function in order to detect and to identify the crack location and the nondimensional crack depth of each crack.

Moreover, it may be observed that the damage assessment technique permits assessment of the number of cracks induced on the beam. In all cases, the identifications of the crack parameters are obtained with satisfactory precisions even if $10 \%$ or $20 \%$ uniformly distributed random noise level is added to the simulations, and only few degrees of freedom are available (less than $5 \%$ of the degrees of freedom of the system).

Finally, the efficiency and robustness of this non destructive detection method are demonstrated through various numerical simulations in regard to the non-dimensional crack depth and the crack location. However, this study demonstrates that the measured data should have a certain level of accuracy in order to allow a correct damage detection.

\section{References}

[1] Friswell, M., Penny, J., and Garvey, S., 1998. "A combined genetic and eigensensitivity algorithm for the location of damage in structures". Computers and Structures, 69, p. 547-556.

[2] Alvandia, A., and Cremona, C., 2006. "Assessment of vibration-based damage identification techniques". Journal of Sound and Vibration, 292, p. 179-202. 
[3] Khiem, N., and Lien, T., 2004. "Multi-crack detection for beam bythe natural frequencies". Journal of Sound and Vibration, 273, p. 175-184.

[4] Ruotolo, R., and Surace, C., 1997. "Damage assessment of multiple cracked beams: numerical results and experimental validation". Journal of Sound and Vibration, 195(3), pp. 456-477.

[5] Kisa, M., and Gurel, M. A., 2006. "Modal analysis of multi-cracked beams with circular cross section". Engineering Fracture Mechanics, 73, p. 963-977.

[6] Chang, C., and Chen, L., 2005. "Detection of the location and size of cracks in the multiple cracked beam by spatial wavelet based approach". Mechanical Systems and Signal Processing, 19, p. 139-155.

[7] Mottershead, J., and Friswell, M., 1993. "Model updating in structural dynamics: a survey". Journal of Sound and Vibration, 167 (2), pp. 347-375.

[8] Baruch, M., 1982. "Optimal correction of mass and stiffness matrices using measured modes". AIAA Journal, 20 (11), pp. 1623-1626.

[9] Berman, A., and Nagy, E., 1983. "Improvement of a large analytical model using test data". AIAA Journal, 21 (8), pp. 1168-1173.

[10] H.Berger, Ohayon, R., Quetin, L., Barthe, L., Ladevèze, P., and Reynier, M., 1991. Updating methods for structural dynamics models : 9-20. La Recherche Aérospatiale 5 (in French).

[11] Farhat, C., and Hemez, F., 1993. "Updating finite element dynamics models using an element-by-element sensitivity methodology”. AIAA Journal, 31 (9), pp. 1702-1711.

[12] Piranda, J., Lallement, G., and Cogan, S., 1991. "Parametric correction of finite element modes by minimization of an output residual: improvement of the sensitivity method". Proc. of the 9th International Modal Analysis Conference.

[13] Lammens, S., Brughmans, M., Leuridan, J., Heylen, W., and Sas, P., 1995. “Application of a frf based model updating technique for the validation of a finite element model of components of the automotive industry”. ASME Conference, Boston, pp. 1191-1200.

[14] Ladevèze, P., and Reynier, M., 1989. "Fe modeling and analysis: a localization method of stiffness errors and adjustments of fe models". Vibrations Analysis Techniques and Application, ASME Publishers.

[15] Ladevèze, P., Nedjar, D., and Reynier, M., 1994. "Updating of finite element models using vibrations tests". AIAA Journal, 32 (7), pp. 1485-1491.

[16] Ladevèze, P., and Chouaki, A., 1999. "Application of a posteriori error estimation for structural model updating". Inverse Problems, 15, pp. 49-58.

[17] Chouaki, A., Ladevèze, P., and Proslier, L., 1998. "Updating structural dynamics models with emphasis on the damping properties". AIAA Journal, 36 (6), pp. 1094-1099.

[18] Friswell, M., 2007. "Damage identification using inverse methods". Philosophical Transactions of the Royal Society A, 365, p. 393-410.

[19] Deraemaeker, A., Ladevèze, P., and Romeuf, T., 2004. "Model validation in the presence of uncertain experimental data". Engineering Computational, 21 (8), pp. 808-833. 
[20] Deraemaeker, A., Ladevèze, P., and Leconte, P., 2002. "Reduced based for model updating in structural dynamics based on constitutive relation error". Computational Methods in Applied Mechanics and Engineering, 191, pp. 2427-2444.

[21] Nelson, H., and Nataraj, C., 1986. "The dynamics of a rotor system with a cracked shaft". Journal of Vibration, Acoustics, Stress and Reliability in Design, 108, p. 189-196.

[22] Davies, W. G. R., and Mayes, I. W., 1984. "The vibrational behaviour of a multi-shaft, multi-bearing system in the presence of a propagating transverse crack". Transactions of the ASME Journal of Vibration, Acoustics, Stress, and Reliability in Design, 106, pp. 146-153.

[23] Mayes, I. W., and Davies, W. G. R., 1984. "Analysis of the response of a multi-rotor-bearing system containing a transverse crack in a rotor". Transactions of the ASME Journal of Vibration, Acoustics, Stress, and Reliability in Design, 106, pp. 139-145.

[24] Sinou, J.-J., and Lees, A. W., 2005. "Influence of cracks in rotating shafts". Journal of Sound and Vibration, 285(4-5), pp. 1015-1037. 